\title{
CARACTERÍSTICAS GRANULOMÉTRICAS DE LOS FONDOS BLANDOS EN UN ÁREA DE LA BAHÍA DE TAGANGA (COLOMBIA) INFLUENCIADA POR LA INSTALACIÓN DE REFUGIOS BENTÓNICOS
}

\begin{abstract}
GRANULOMETRIC CHARACTERISTICS OF THE SOFT BOTTOMS IN AN AREA OF THE TAGANGA BAY (COLOMBIA) INFLUENCED BY THE INSTALATION OF BENTONIC SHELTERS
\end{abstract}

\author{
Juan Carlos Rubio-Polanía ${ }^{1}$, Carlos Alberto Trujillo-Arcila ${ }^{2}$
}

\begin{abstract}
${ }^{1}$ Biologo Marino, Estudiante de Maestría en Ciencias, CICESE. Calle 179 No 6-64 apto 503, Bogotá D.C, Colombia. E-mail: jucarupo2@hotmail.com; ${ }^{2}$ Biólogo Marino, grupo de Ciencias Marinas Aplicadas al Desarrollo Integrado de Zonas Costeras adscrito a la Fundación Sila Kangama. Carrera 3 No 25-81, Rodadero, Santa Marta, Colombia. ecoreef.colombia@gmail. com
\end{abstract}

Rev. U.D.C.A Act. \& Div. Cient. 16(1): 193 - 203, 2013

\section{RESUMEN}

Los sedimentos marinos y sus características granulométricas pueden condicionar el establecimiento de las comunidades bentónicas. Con el objetivo de establecer el tipo de sedimento presente en el área de influencia de los refugios tipo casita cubana instalados en la bahía de Taganga, la Fundación Sila Kangama, durante el primer semestre de 2004, evaluó las características granulométricas y químicas de los fondos blandos presentes en el área de influencia, de tres de los 15 refugios bentónicos, ubicados a 25 m de profundidad. Se determinó la naturaleza arenosa del sedimento, el cual, fluctuó entre arenas finas, medias y gruesas, con mezcla de material fino y grueso, sometido a una energía cinética media alta, con clasificaciones de moderadas a muy malas, que están en relación con valores de asimetría negativos y distribuciones platicúrticas, que indican bimodalidad. Los porcentajes de materia orgánica calculados son atípicos (entre 9,99 y 23,66) para sedimentos arenosos y la característica bioclástica resulta de la descomposición de los restos calcáreos de origen biológico. Se observaron dos tipos de ambientes sedimentarios, de erosión en la estación uno y de flujo, en las estaciones dos y tres, lo cual, se vio reflejado en las pruebas no paramétricas de Kruskall-Wallis y Dunn realizadas. No se observa un efecto marcado de los refugios bentónicos sobre los sedimentos evaluados.

Palabras clave: Sedimentos, granulometría, Bahía Taganga, Caribe colombiano.

\section{SUMMARY}

The establishment of benthic communities depends on the granulometric characteristics of marine sediments. The main objective of this study was to establish the kind of sediment present in the area of influence of casita cubana shelters installed in the Taganga Bay, the Sila Kangama foundation during the first semester of 2004, evaluating the granulometric characteristics and the chemistry of soft bottom present in the area influenced by three of 15 benthic refuges installed in $25 \mathrm{~m}$ depth in the interior of the bay. The nature of the sandy sediments, fluctuated between fine, medium and coarse, with a mixture with fine and coarse materials, under medium to high kinetic energy, with a classification ranging from moderate to poor relating to the negative asymmetry values and platikutic distributions indicating bimodality. The percentages of organic material calculated are atypical (between 9.99 and 23.66) for sandy sediments and its bioclastic characteristics are the result of the decomposition of calcareous fragments of biological origin. Two types of sediment environments were observed, one of erosion at station one and one of water flow in stations two and three, which were reflected in the Kruskall-Wallis and Dunn nonparametric tests carried out. No marked effect was observed of the benthic refuges on the sediments evaluated.

Key words: Sediments, Granulometry, Taganga Bay, Colombian Caribbean. 


\section{INTRODUCCIÓN}

Los sedimentos marinos son agregados de materiales insolubles no consolidados de origen orgánico e inorgánico, llevados a los fondos costeros y oceánicos por agentes de transporte (Ruíz \& Ilin, 1979). Estos son importantes en el desarrollo de cadenas tróficas, reciclamiento de materia orgánica, equilibrio atmósfera-océano, estudios geológicos y evaluaciones de impacto ambiental (De la Lanza, 1986; Valdés et al. 2000). Sus características texturales pueden condicionar el establecimiento de las comunidades bentónicas (Wieser, 1969).

El análisis de tamaño de grano es una herramienta indispensable para clasificar ambientes sedimentarios (Blott \& Pye, 2001), que son sustratos móviles, influenciados por procesos físicos, químicos y biológicos (Sebens, 1991). Su distribución y baja complejidad estructural facilita la disposición de hábitats artificiales, que favorecen la agregación de especies de importancia ecológica y comercial (Vegas, 1971; Vita et al. 2002; Delgadillo-Garzón, 2009). Tal es el caso de los refugios bentónicos, llamados casitas cubanas, ubicados en la bahía de Taganga (Figura 1), que se encuentran entre los 20 y 30m de profundidad, distribuidos en un área de 1000 $\mathrm{m}^{2}$. Estas estructuras fueron dispuestas por la Fundación Sila Kangama, para realizar la evaluación del reclutamiento y mejorar el manejo de la langosta espinosa, en el área de Santa Marta (Jaimes et al. 2004) y son ampliamente utili- zados en la explotación de la langosta espinosa (Panulirus argus, Latreille, 1804), en la cuenca del caribe (Arango et al. 2001).

La bahía de Taganga, ubicada al Norte de la ciudad de Santa

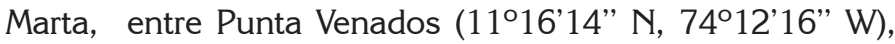
Punta Colorada ( $11^{\circ} 15^{\prime} 44^{\prime \prime} \mathrm{N}, 74^{\circ} 11^{\prime} 56^{\prime \prime}$ W) y la playa de Taganga (74¹1'29" W), (Figura 2) está formada por estribaciones de la Sierra Nevada de Santa Marta y presenta una variedad de hábitats, que van desde formaciones coralinas hasta fondos sedimentarios (Agenda Caribe, 2006).

La bahía presenta litorales rocosos, escarpados por esquistos verde azulados de foliación metamórfica y batolitos, con muchas puntas y bahía. Las playas son arenosas, con abundantes cascajos de naturaleza litológica y carbonato de calcio de origen coralino. Los fondos rocosos son frecuentes en el área, debido a la presencia de las estribaciones noroccidentales de la Sierra Nevada de Santa Marta (Bartels, 1970; Bustamante et al. 2008).

Este trabajo, se realizó con el objetivo de contribuir y de fomentar el conocimiento de los fondos sedimentarios de la bahía de Taganga, teniendo en cuenta que es un área turística importante del caribe colombiano, que se encuentra sometida a factores estresantes de tipo antropogénico (e.g. descargas del emisario submarino).

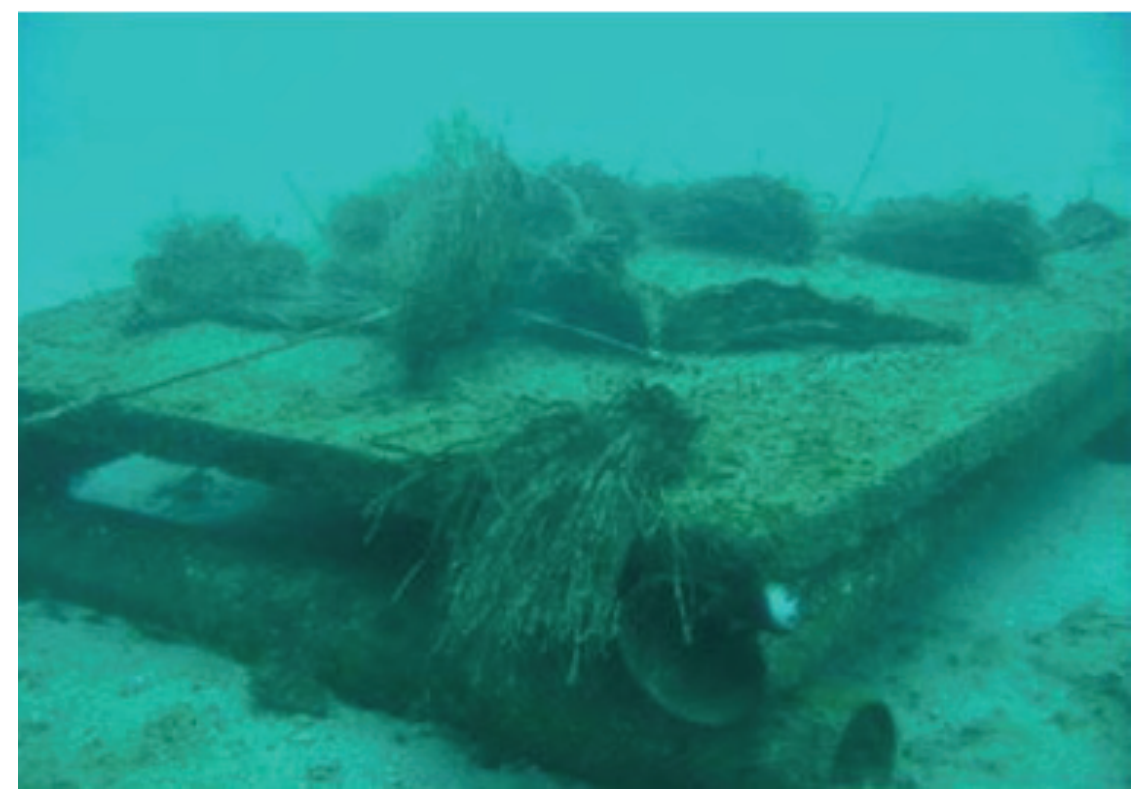

Figura 1. Casita Cubana dispuesta en la bahía de Taganga. 


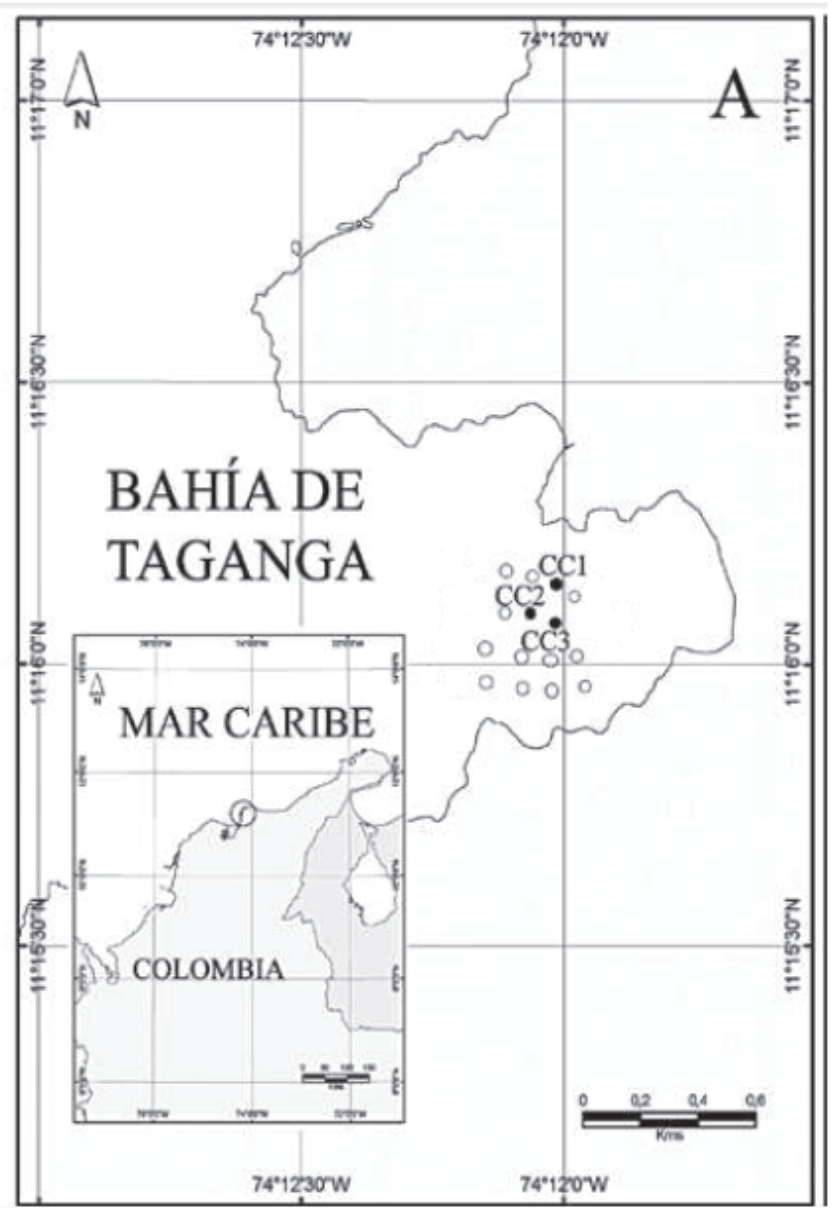

Figura 2. Mapa de la bahía de Taganga, se muestran los tres refugios bentónicos muestreados (CC1, CC2 y CC3) (tomado y modificado de Delgadillo-Garzón, 2009).

\section{MATERIALES Y MÉTODOS}

Se seleccionaron de manera aleatoria tres refugios bentónicos, con el fin de tener una muestra representativa del área. Cada estructura fue numerada y geoposicionada, de tal manera que la estación número uno, se encuentra ubicada a $11^{\circ} 15 ’ 56,5^{\prime \prime}$ N y $74^{\circ} 11^{\prime} 51,1^{\prime \prime}$ W; la estación dos, a $11^{\circ} 15^{\prime} 57,9^{\prime \prime}$ N y $74^{\circ} 11^{\prime} 51,7^{\prime \prime} \mathrm{W}$ y, la tres, a $11^{\circ} 15^{\prime} 57,5^{\prime \prime} \mathrm{N} \mathrm{y}$ $74^{\circ} 11^{\prime} 51,0^{\prime \prime} \mathrm{W}$. La toma de muestras en cada estructura, se realizó sobre un transecto, de $10 \mathrm{~m}$ con dirección Norte-Sur.

Para cada unidad muestreal fueron tomadas dos réplicas: la primera destinada para el análisis granulométrico y, la segunda, para el análisis químico. El sedimento fue extraido usando equipo autónomo de buceo y una caja excavadora de $0,02 \mathrm{~m}^{2}$ (con una máxima profundidad de perforación de $30 \mathrm{~cm}$ ), colectándose el sedimento a $5 \mathrm{~m}, 2 \mathrm{~m}$ y debajo de la estructura $(0 \mathrm{~m})$ (Figura 3); el material extraído fue almacenado en bolsas rotuladas. Muestras de cinco centímetros de espesor, destinadas para medir el contenido de materia orgánica, fueron almacenadas en frascos plásticos negros, los cuales, se mantuvieron congelados hasta el momento de hacer la determinación.

Para el análisis granulométrico, se tomó $100 \mathrm{~g}$ de sedimento, previamente secados y homogenizados, que fueron tamizados durante 15 minutos, en un vibrador Retsch, haciendo pasar la muestra sobre una columna de tamices con luces de malla de $4 ; 2 ; 1,45 ; 0,635 ; 0,45 ; 0,35 ; 0,160 ; 0,112 ; 0,063$ y $<0,063 \mathrm{~mm}$. A continuación, se pesó el contenido de cada tamiz y se transformó a porcentaje (Buchanan, 1984), se calcularon cinco características texturales, que son, según Bartholdy et al. (2007), las que mejor reflejan las tendencias de los parámetros texturales. 1) la Mediana $(M d=\varphi 50)$, a los efectos de estimar la energía cinética media (ECM) del ambiente sedimentario; 2) El tamaño gráfico promedio o Media $(M z=\{(\varphi 16+\varphi 50+\varphi 84) / 3\})$, para establecer el diámetro promedio de las partículas de la muestra; 3 ) La Desviación 


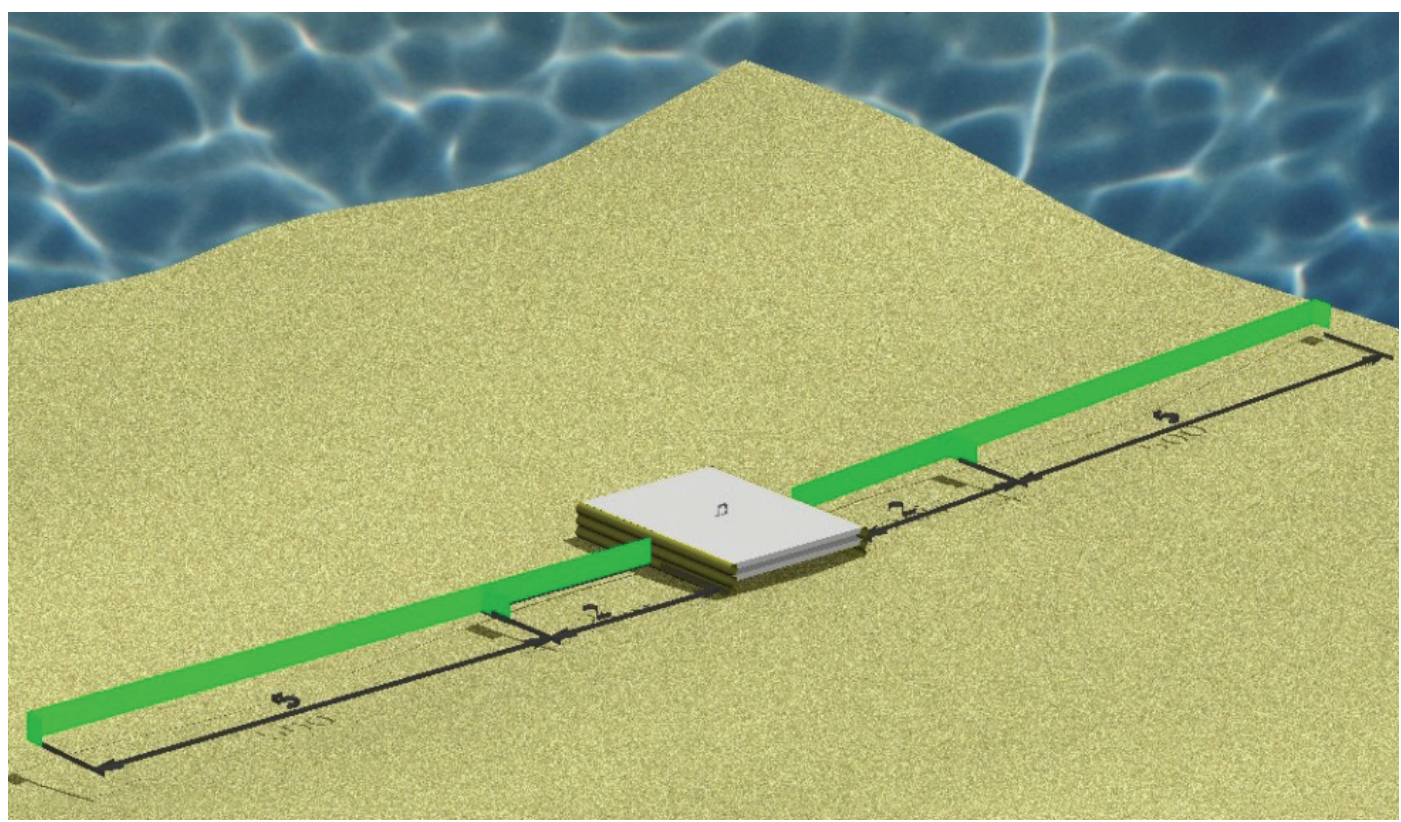

Figura 3. Diagrama de los cinco puntos de muestreo: cuatro externos y uno debajo del refugio (las distancias de muestreo están en metros). La figura rectangular gris representa el refugio bentónico.

estándar gráfica inclusiva $\left(\sigma_{1=}\{(\varphi 84+\varphi 16) / 4\}+\{(\varphi 95-\right.$ $\varphi 5)$ / 6.6\}), por medir la clasificación o uniformidad del sedimento; 4) Asimetría gráfica inclusiva (Sk $=\{\varphi 16+\varphi 84-2$ $\varphi 50 / 2(\varphi 84-\varphi 16)\}+\{\varphi 5+\varphi 95-2 \varphi 50 / 2(\varphi 95-\varphi 5)\})$, para observar el sesgo que presenta la curva de distribución hacia granos finos o gruesos y, 5) La cúrtosis gráfica $(\mathrm{Kg}=$ $\{(\varphi 95-\varphi 5) / 2.44(\varphi 75-\varphi 25)\})$, para establecer el acercamiento al valor medio de la curva de distribución (Méndez et al. 1986; García et al. 2009). Todos los cálculos se realizaron conforme al trabajo de Folk \& Ward (1957).

El porcentaje de materia orgánica, se determinó por pérdida de peso por ignición, sometiendo cada muestra por duplicado, en alícuotas de $1 \mathrm{~g}$ de peso, a un secado de $95^{\circ} \mathrm{C}$, durante 24 horas. Posteriormente, se calcinaron las muestras a $550^{\circ} \mathrm{C}$, por una hora y se calculó la diferencia en peso (Páez-Osuna et al. 1984).

Para la determinación de carbonatos totales, se tomó una muestra de $10 \mathrm{~g}$ de sedimento, a los cuales, se les agregó $50 \mathrm{~mL} \mathrm{HCl} 0,5 \mathrm{~N}$; posteriormente, la muestra fue calentada durante 5 min y, luego, titulada con $\mathrm{NaOH} 0,25 \mathrm{~N}$, usando fenolftaleína, como indicador. Con el volumen utilizado en la titulación, se calcula la cantidad de carbonatos presentes en la muestra, que son expresados como porcentaje (Hernández, 1994).

Debido a que los datos no cumplieron los supuestos de nor- malidad y de homogeneidad de varianza, se realizaron las pruebas no paramétricas de Kruskall-Wallis y de Dunn, con los paquetes estadísticos Statgraphics Centurión XVI y Biostat, con el fin de determinar la existencia de diferencias estadísticamente significativas entre las tres estaciones.

\section{RESULTADOS Y DISCUSIÓN}

El sedimento hallado en las tres estaciones está constituido por arenas de color gris, con una elevada presencia de restos orgánicos calcáreos, provenientes de moluscos, de crustáceos y de foraminíferos. Los valores de tamaño medio de grano (Mz), ubican al sedimento analizado en el intervalo de las arenas finas, arenas medias y arenas gruesas (Tablas 1 y 2). Esta textura sedimentaria, se encuentra con regularidad en el interior de la plataforma continental (Gilluly et al. 1964) y es reportada por Von (1976), Gaitán-Espitia (2008) y Martínez-Ramírez et al. (2009), en la bahía de Taganga.

El predominio de la textura arenosa señala la presencia de una alta energía cinética media (ECM), generada por el continuo movimiento turbulento de agua, que favorece la depositación de material grueso y arrastra el material fino, impidiendo su asentamiento (Edward \& Héquette, 2007; Guinez et al. 2010); esta situación es confirmada por los valores de mediana (Md) (Tabla 1 y 2). Estos resultados concuerdan con lo observado por Ramírez-Barón et al. (2010), quienes establecen la presencia de aguas turbulentas en la bahía de 
Tabla 1. Rangos de los parámetros texturales calculados: mediana (Md); tamaño medio de grano o Media (Mz); desviación estándar $\left(\sigma_{1}\right)$; cúrtosis $(\mathrm{kg})$ y asimetría $(\mathrm{Sk})$.

\begin{tabular}{|c|c|}
\hline \multicolumn{2}{|c|}{ Diámetro Medio o Mediana (Md) } \\
\hline Arenas muy gruesas & $-1,0 \varphi$ а $0 \varphi$ \\
\hline Arenas gruesas & $0,01 \varphi$ а $1,0 \varphi$ \\
\hline Arenas medias & $1,01 \varphi$ a $2.0 \varphi$ \\
\hline Arenas finas & $2,01 \varphi$ а $3,0 \varphi$ \\
\hline Arenas muy finas & $3,01 \varphi$ a $4,0 \varphi$ \\
\hline \multicolumn{2}{|c|}{ Tamaño Gráfico Promedio o Media (Mz) } \\
\hline Grava & $-2 \varphi$ а $-0,99 \varphi$ \\
\hline Arena muy gruesa & $-1 \varphi$ а $0 \varphi$ \\
\hline Arena gruesa & $0,01 \varphi$ а $1,0 \varphi$ \\
\hline Arena media & $1,01 \varphi$ a $2,0 \varphi$ \\
\hline Arena fina & $2,01 \varphi$ а $3,0 \varphi$ \\
\hline Arena muy fina & $3,01 \varphi$ а $4,0 \varphi$ \\
\hline Arcillas y Limos & $4,0 \varphi$ a $14 \varphi$ \\
\hline \multicolumn{2}{|c|}{ Desviación Estándar Gráfica Inclusiva o clasificación $\left(\sigma_{1}\right)$} \\
\hline Muy bien Clasificado & $\sigma_{1}$ menor a $0,35 \varphi$ \\
\hline Bien clasificado & $0,35 \varphi$ а $0,50 \varphi$ \\
\hline Moderadamente bien clasificado & $0,50 \varphi$ а $0,71 \varphi$ \\
\hline Moderadamente clasificado & $0,71 \varphi$ а $1,00 \varphi$ \\
\hline Mal clasificado & $1,00 \varphi$ а $2,00 \varphi$ \\
\hline Muy mal clasificado & $2,00 \varphi$ а $4,00 \varphi$ \\
\hline Extremadamente mal clasificado & Mayor a $4,00 \varphi$ \\
\hline \multicolumn{2}{|c|}{ Asimetría Gráfica Inclusiva (Sk) } \\
\hline Muy asimétricos hacia los tamaños finos & $1,00 \varphi$ а $0,30 \varphi$ \\
\hline Asimétricos hacia los tamaños finos & $0,30 \varphi$ а $0,10 \varphi$ \\
\hline Casi simétrico & $0,10 \varphi$ a $-0,10 \varphi$ \\
\hline Asimétricos hacia los tamaños gruesos & $-0,10 \varphi$ а $-0,30 \varphi$ \\
\hline Muy asimétricos hacia los tamaños gruesos & $-0,30 \varphi$ a $-1,00 \varphi$ \\
\hline \multicolumn{2}{|c|}{ Cúrtosis Gráfica (kg) } \\
\hline Muy Platicúrtica & kg menor a $0,67 \varphi$ \\
\hline Platicúrtica & $0,67 \varphi$ а $0.90 \varphi$ \\
\hline Mesocúrtica & $0.90 \varphi$ а $1,11 \varphi$ \\
\hline Leptocúrtica & $1,11 \varphi$ а $1,50 \varphi$ \\
\hline Muy leptocúrtica & $1,50 \varphi$ а $3,00 \varphi$ \\
\hline Extremadamente leptocúrtica & Menor a $3,00 \varphi$ \\
\hline
\end{tabular}


Tabla 2. Valores de los parámetros texturales calculados, materia orgánica (M.O), carbonato de calcio $\left(\mathrm{CaCO}_{3}\right)$, tamaño medio de grano $(\mathrm{Mz})$, cúrtosis gráfica $(\mathrm{kg})$, desviación estándar $\left(\sigma_{1}\right)$ y asimetría $(\mathrm{Sk})$.

\begin{tabular}{|c|c|c|c|c|c|c|c|c|}
\hline Refugio & $\begin{array}{l}\text { Punto de } \\
\text { Muestreo }\end{array}$ & Md & $M z$ & $\sigma_{1}$ & Sk & kg & \% M.O & $\% \mathrm{CaCO} 3$ \\
\hline \multirow{5}{*}{ 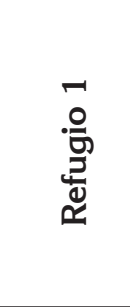 } & $5 \mathrm{~m} \mathrm{~N}$ & 2,75 & 1,80 & 1,79 & $-0,71$ & 0,86 & 12,89 & 99,70 \\
\hline & $2 \mathrm{~m} \mathrm{~N}$ & 1,95 & 1,58 & 1,84 & $-0,28$ & 1,05 & 17,22 & 98,60 \\
\hline & $0 \mathrm{~m}$ & 2,85 & 2,02 & 1,73 & $-0,57$ & 0,83 & 21,01 & 99,70 \\
\hline & $2 \mathrm{~m} \mathrm{~s}$ & 2,80 & 1,91 & 1,67 & $-0,77$ & 0,92 & 16,81 & 99,30 \\
\hline & $5 \mathrm{~m} \mathrm{~S}$ & 1,89 & 1,50 & 1,74 & $-0,32$ & 0,85 & 19,70 & 99,70 \\
\hline \multirow{5}{*}{ 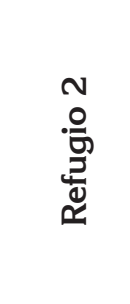 } & $5 \mathrm{~m} \mathrm{~N}$ & 1,10 & 0,87 & 1,97 & $-0,12$ & 0,73 & 15,16 & 99,10 \\
\hline & $2 \mathrm{~m} \mathrm{~N}$ & 1,40 & 1,23 & 1,78 & $-0,15$ & 1,44 & 17,08 & 99,10 \\
\hline & $0 \mathrm{~m}$ & 1,70 & 1,45 & 1,71 & $-0,16$ & 1,03 & 23,66 & 97,90 \\
\hline & $2 \mathrm{~m} \mathrm{~S}$ & 0,90 & 0,67 & 1,86 & $-0,11$ & 0,87 & 8,49 & 97,20 \\
\hline & $5 \mathrm{~m} \mathrm{~S}$ & 0,35 & 0,58 & 1,61 & 0,19 & 0,86 & 19,58 & 99,30 \\
\hline \multirow{5}{*}{ 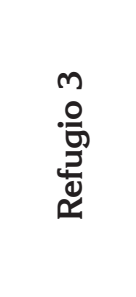 } & $5 \mathrm{~m} \mathrm{~N}$ & 1,50 & 1,22 & 1,94 & $-0,17$ & 0,86 & 14,66 & 99,00 \\
\hline & $2 \mathrm{~m} \mathrm{~N}$ & 0,65 & 0,07 & 2,31 & 0,44 & 0,55 & 9,99 & 98,90 \\
\hline & $0 \mathrm{~m}$ & 1,00 & 0,96 & 2,08 & 0,00 & 0,68 & 14,38 & 99,10 \\
\hline & $2 \mathrm{~m} \mathrm{~S}$ & 1,60 & 0,93 & 2,29 & $-0,29$ & 0,73 & 21,51 & 99,20 \\
\hline & $5 \mathrm{~m} \mathrm{~S}$ & 1,46 & 1,23 & 1,94 & $-0,10$ & 0,81 & 17,72 & 98,50 \\
\hline
\end{tabular}

Taganga, en una estación ubicada a 1,43km mar afuera de las tres estaciones.

La desviación estándar gráfica inclusiva $\left(\sigma_{1}\right)$ fluctuó entre $1,61 \varphi$ y $2,31 \varphi$, valores que indican una mala y muy mala clasificación de los sedimentos hallados. De acuerdo a Folk \& Ward (1957), las arenas finas y medias registradas en nuestro estudio presentan una buena clasificación; sin embargo, la mezcla de partículas finas $(2,01 \varphi$ a $3,00 \varphi)$ con partículas gruesas $(-2,0$ y $1,0 \varphi)$ genera un aumento en los valores de desviación estándar, siendo estos ambientes sedimentarios heterogéneos (Folk \& Ward, 1957; Héquette et al. 2008).

De los 15 puntos de muestreo evaluados, 12 presentaron valores negativos de asimetría, que se relaciona con la mala y muy mala clasificación del sedimento (Gao et al. 1994; Parrado \& Achab, 1999), debido a que la mezcla de material fino y grueso genera una distribución semejante a la de chi cuadrado, lo cual, se observa como un movimiento de la media y la moda al interior de la curva de distribución gráfica hacia la izquierda, haciéndola decrecer (Emeka et al. 2010), lo que indica, que el traslado de los granos finos presentes en la cola de distribución se da por selección de partículas ante una energía cinética media más alta de lo normal (en términos de intensidad y duración), con turbulencia y adiciones de restos calcáreos gruesos. Estos últimos son una población subordinada al interior de la muestra, que permiten presumir que en el área se dan procesos de erosión (Figura 4) (Folk, 1966; Martins, 2003; Emeka et al. 2010).

Los valores de asimetría positiva observada en dos puntos de muestreo, uno ubicado en la estación dos y uno en la estación tres (Tabla 2), señalan la presencia de poblaciones dominantes gruesas con adición de material fino, con competencia de agentes de transporte y la existencia de procesos de depositación (Martins, 2003). Esto se observa en ambientes en estado de flujo, donde existen procesos de erosión y de depositación (Martins, 2003).

En los 15 puntos de muestreo, se observaron cuatro tipos de curvas de distribución, que van desde muy platicúrtica (aplanada) hasta leptocúrtica (Tabla 2). Las curvas de distribución platicúrticas resultan de la presencia de muchas clases de tamaños de grano, que presentan valores similares con me- 
jor clasificación en los extremos, por lo que este ambiente es poco estable (Méndez et al. 1986; Amrouni-Bouaziz et al. 2007). Esta condición se observó en el $73 \%$ del total muestreado e indica la bimodalidad de los sedimentos en la curva de distribución (Figura 4); esta situación se suele presentar en sedimentos con mezcla de materiales finos y gruesos con mala clasificación (Folk \& Ward, 1957).
Un punto de muestreo ubicado en la estación dos (Tabla 1 y 2) presentó leptocúrtosis, tipo de distribución, en el que la selección de partículas del centro de la curva de distribución es mejor que en los extremos (García et al. 2009). La acumulación en el centro de la curva es un indicador de la presencia de una ECM alta, que dura más tiempo de lo normal (Martínez, 1986).

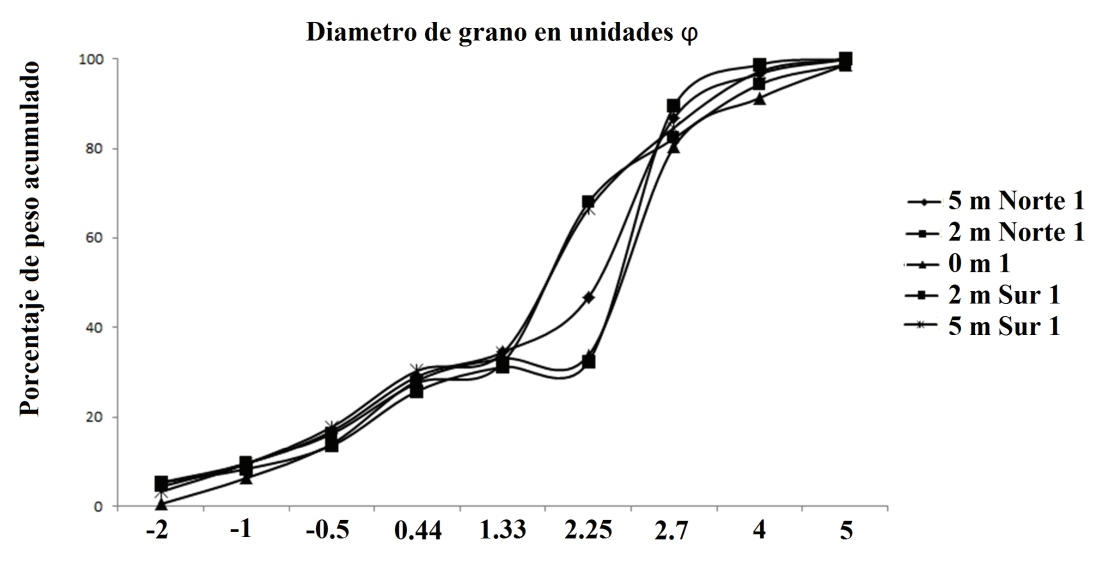

Diametro de grano en unidades $\varphi$

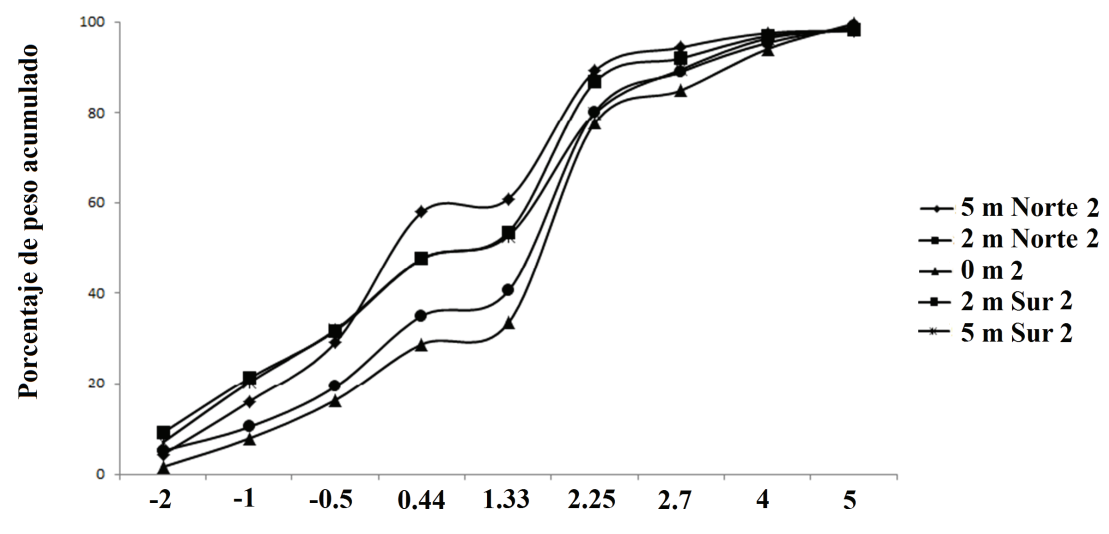

Diametro de grano en unidades $\varphi$

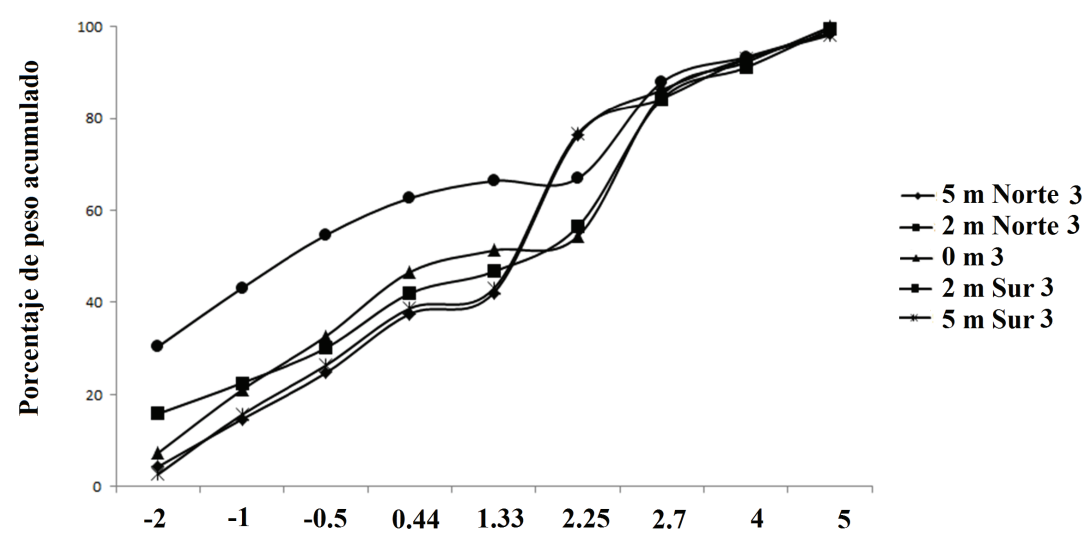

Figura 4. Curvas de porcentaje de peso acumulado vs. diámetro en unidades phi $(\varphi)$, de las tres estaciones. 
En la estación tres, se observó el único valor extremo de cúrtosis (Tabla 2) (muy platicúrticas), que indica que las partículas han sido transportadas en ambientes de alta ECM sin que sufran grandes modificaciones en el tamaño de grano; se observan modas subiguales (Folk \& Ward, 1957).

Se percibió mesocúrtosis en tres puntos de muestreo (Tabla 1 y 2); en este caso, las distribución de partículas fue muy cercana a la normal, es decir, que no hubo oscilaciones en la ECM en la región central de la curva de distribución (Fuentes, 2006).

Los porcentajes de materia orgánica calculados (Tabla 2) son atípicos en sedimentos arenosos, que presentan espacios intersticiales, que favorecen la depositación de agua que oxida el material orgánico y el estado libre de las bacterias, encargadas de los procesos diagénicos (INVEMAR, 2003; De la Lanza, 1986). Esto indica, que en el área existen otras fuentes de material orgánico, además de la producción primaria y el material erosionado y lixiviado no degradado, en la columna de agua (Ruíz \& Ilin, 1979).

En áreas perturbadas, como el golfo de Vizcaya, Martínez \& Adarraga (2001) reportan 2,37\% de materia orgánica en arenas gruesas y $1,82 \%$, en arenas finas y Vita et al. (2002) calcularon un máximo de $8 \%$, para un área expuesta a la influencia de jaulas de explotación de acuicultura, siendo los valores reportados por ambas investigaciones más bajos que los calculados para la bahía de Taganga; esto puede ser el resultado de la suma de factores como: 1) la disminución de permeabilidad, debida a la mezcla de arenas y de gravas, que afecta la penetración de agua en espacios intersticiales (Visher, 1969), que resulta de un ambiente reductor, donde se preserva la materia orgánica (evidenciado en el color gris) (Lamas, 2006); 2) aportes provenientes del emisario submarino, que pueden ser transportados al interior de la bahía, durante el segundo semestre del año, cuando se produce un transporte de las masas de agua hacia el interior de la bahía (Capitanía de Puerto, 1995; Agenda Caribe, 2006) y, 3) a la actividad de organismos asociados a los refugios bentónicos (Fabi et al. 2002), debido a que estos refugios concentran crustáceos, moluscos, anélidos, esponjas, equinodermos y especies ícticas (Arango et al. 2001; Delgadillo-Garzón, 2009), factores responsables del elevado porcentaje de materia orgánica.

El porcentaje de carbonato de calcio fluctuó entre los 97,20\% y $99,70 \%$, lo que establece la naturaleza bioclástica del sedimento analizado (Tabla 3). Vegas (1971) y Arche (1992) establecen que las arenas de los márgenes continentales están constituidas, principalmente, por cuarzos procedentes de la erosión continental y de detritos calcáreos provenientes de valvas de moluscos, caparazones de crustáceos y de equinodermos, de escamas, de huesos de peces y de restos coralinos.
Dichos restos, pertenecientes a la tanatocenosis, fueron reportados en la bahía de Taganga en la isobata de $16 \mathrm{~m}$, a profundidades entre los 25 y 30m y a 70m de la línea costa (Von, 1976; Díaz, 1990; Martínez-Ramírez et al. 2009) y se mantuvieron presentes, de manera abundante y generalizada, en los sedimentos analizados; los mismos fueron los responsables del elevado porcentaje de carbonato de calcio hallado en las muestras (Salazar et al. 2003) y una clara evidencia de la sedimentación organógena presente en la bahía (Hernández et al. 2002).

La prueba no paramétrica de Kruskall-Wallis indicó diferencias estadísticamente significativas entre las estaciones $(\mathrm{H}=$ $6,17 p<0=0,009$ ), mientras que la prueba a posteriori de Dunn, demostró que las estaciones dos y tres no presentan diferencias estadísticamente significativas, con un valor $Q$ de 0,0 , mientras que entre la estación uno y las estaciones dos y tres se presenta diferencias estadísticamente significativas (con un valor de $Q$ calculado fue de 2,65), siendo esta diferencia un reflejo de la presencia de una ECM más elevada en las estaciones dos y tres, sumado al estado de flujo que presentan estas dos estaciones.

Según Fabi et al. (2002) y Vita et al. (2002), las estructuras artificiales generan efectos sobre la dinámica sedimentaria, donde se disponen (e.g. aumento en el contenido de materia orgánica, distribución del sedimento o cambios en el tamaño de grano); sin embargo, esto no se observó un efecto marcado de los refugios sobre la disposición y la distribución de los fondos sedimentarios circundantes, debido a que solamente en las estaciones uno y dos fue evidente la disminución en la ECM debajo de los refugios, sumado a valores más altos de materia orgánica y el aumento de la ECM desde el refugio hacia los puntos de muestreo, ubicados en el sur. Por lo tanto, resulta necesario realizar investigaciones tendientes a evaluar la cantidad y la tasa de enriquecimiento orgánico, así como las características texturales de toda la bahía y una evaluación amplia del total de refugios dispuestos, tanto en la bahía de Taganga, como en el área de Pozos Colorados.

Agradecimientos. Los autores agradecen a Jagannath, Rubén Darío Rubio Ospina, Yolanda Polanía Serrano, Juan Pablo Valencia Vera, Óscar Delgadillo, Johanna Medellín, Lina Ceballos, Claudia Catalina Barón, Bibiana Molina, Aminta Jauregui, Paulo César Tigreros Benavides, Ana Uribe, Rafael Barragán y Camila Di marco. Conflicto de intereses: Los autores del presente escrito declaran que no existe conflicto de intereses que ponga en riesgo la validez de los resultados. Financiación: Esta investigación fue financiada por la Fundación Sila Kangama y el Fondo para la Acción Ambiental, dentro del proyecto "Variabilidad del asentamiento post larval e implementación de refugios artificiales para el manejo de la langosta espinosa Panulirus argus en la región de Santa Marta" y recibió el apoyo de la Universidad Jorge Tadeo Lozano. 


\section{BIBLIOGRAFÍA}

1. AGENDA CARIBE. 2006. http://agendacaribe.blogspot. mx/2006/09/grave-amenaza-la-vida-y-al-medio.html (con acceso 27/10/2011).

2. ARCHE, A. 1992. Sedimentología. Vol.1. Madrid, España. 258p.

3. AMROUINI-BOUAZIZ, O.; SOUISSI, R.; BARUSSEAU, J.P.; ABDELJAOUED, S.; PAUC, H.; CERTAIN, R. 2007. Grain-size and morphodynamical state of the bay-of-Mahdia shoreface (Tunisia). Contribution to the assessment of coastal sensitivity. Geo-Eco-marina (Rumania). 13:5-19.

4. ARANGO, L.; MANRIQUE, M.; JAIMES, J. 2001. Reclutamiento de juveniles de la langosta Panulirus argus con refugios artificiales en el Caribe colombiano. Rev. Invest. Marinas (Cuba). 22(3):221-228.

5. BARTELS, G. 1970. Geomorphologische Höhenstufen der Sierra Nevada de Santa Marta (Kolumbien). Giessener Geogr. Schr. (Alemania) 21:1-56.

6. BARTHOLDY, J.; CHRISTIANSEN, C.; PEDERSEN, J. 2007. Comparing spatial grain-size trends inferred from textural parameters using percentile statistical parameters and those based on the log-hyperbolic method. Sediment. Geology. (Paises Bajos). 202:436-452.

7. BLOTT, S.J.; PYE, K. 2001. Gradistat: A grain size distribution and statistics package for the analysis of unconsolidated sediments, Earth Surf. Process. Landf (Inglaterra). 26:1237-1248.

8. BUCHANAN, J. 1984. Sediment Analysis. En: Holme, N. y McIntyre A. Methods for the Study of Marine Benthos. Ed. Blackwell Scientific Publication. (Reino Unido). p.41-63.

9. BUSTAMANTE, C.; CARDONA, A.; SALDARRIAGA, M.; GARCÍA-CASCO, A.; VALENCIA, V.; WEBER, M. 2008. Metamorfosis de los esquistos verdes y anfibolitas pertenecientes a los esquistos de santa marta, sierra nevada de Santa marta (Colombia): cंregistro de la colisión entre el arco Caribe y la margen suramericana?. Bol. Ciencias Tierra (Colombia). 25:7-25.

10. CAPITANÍA DE PUERTO. 1995. Informe para la implementación de una planta de tratamiento en la bahía de Taganga. Informe técnico, Capitanía de Puerto, Santa Marta. 15p.
11. DE LA LANZA, G. 1986. Materia orgánica en los sedimentos del sistema lagunar Huizache y Caimanero: importancia, comportamiento y significado en modelos de predicción. An. Inst. Mar y Limnol. Univ. Nal. Auntón. México (México). 13(1):251-286.

12. DELGADILlO-GARZÓN, O. 2009. Nota: Peces y macroinvertebrados móviles de hábitats artificiales en la bahía de Taganga, caribe Colombiano. Bol. Invest. Mar. Cost. (Colombia). 38(1):197-204.

13. DÍAZ, J. 1990. Estudio ecológico integrado de la zona costera de Santa Marta y Parque Nacional Natural Tayrona. Programa de ecosistemas marinos INVEMAR. (Colombia). 54p.

14. EDWARD, J.; HÉQUETTE, A. 2007. The grain-size characterization of coastal sand from the Somme estuary to Belgium: Sediment sorting processes and mixing in a tide- and storm-dominated setting. Sediment. Geology. 202:369-382.

15. EMEKA, N.C.; ANTIA, V.; UKPONG, A.; AMAH, E.; NTEKIM, E. 2010. A Study on the Sedimentology of Tidal Rivers: Calabar and Great Kwa, S. E. Nigeria. Eur. J. Sci. Res. (Seychelles). 47(3):370-386.

16. FABI, G.; LUCCARINI, F.; PANFILI, M.; SOLUSTRI, C.; SPAGNOLO, A. 2002. Effects of an artificial reef on the surrounding soft-bottom community (central Adriatic Sea). J. Marine Sci. (Dinamarca). 59(1):343349.

17. FOLK, R. 1966. A review of grain-size parameters. Sedimentology. (Inglaterra). 6:73-93.

18. FOLK, R.; WARD, W. 1957. Brazos River Bar- a study in the significance of grain size parameters. J. Sediment. Petrology. (Estados Unidos). 27:3-27.

19. FUENTES, M. 2006. Algunas características sedimentológicas del puerto pesquero internacional de Güiria y sus alrededores, estado de Sucre, Venezuela. Saber. Univ. De Oriente. (Venezuela). 1(2):197-206.

20. GAITÁN-ESPITIA, J. 2008. Estructura de la comunidad del Phylum Echinodermata en aguas someras de la bahía de Taganga, Caribe colombiano. Rev. U.D.C.A Act. \& Div. Cient. (Colombia). 11(1):85-93.

21. GAO, S.; COLLINS, M.; LANCKNEUS, J.; DE MOOR, G.; VAN LANCKER, V. 1994. Grain size trends associated with transport patterns an example from the 
Belgican continental shelf. Mar. Geolo. (Paises Bajos) 121:171-185.

22. GARCÍA, C.; CASTILLO, R.; GARCÍA, R. 2009. Variación granulométrica y mineralógica en profundidad de los sedimentos retenidos por diques, estudio de cauces torrenciales semiáridos del sureste peninsular. Nimbus. (España). 23-24.

23. GILLULY, J.; WATERS, A.; WOODFORD, A. 1964. Principios de Geología. Aguilar, S.A. 693 p.

24. GUINEZ, M.; VALES, J.; SIFFEDINE, A. 2010. Variabilidad espacial y temporal de la materia orgánica sedimentaria, asociada a la Zona de Mínimo Oxígeno (ZMO), en un ambiente costero del norte de la corriente de Humboldt, bahía de Mejillones, Chile. Lat. Am. J. Aquat. Res (Chile). 38(2):242-253.

25. HERNÁNDEZ, C.; MARTIN, R.; LUQUE, A. 2002. Rasgos sedimentológicos de los fondos marinos de Maspalomas (Gran Canaria). Vegueta. (España). 6:191-200.

26. HERNÁNDEZ, C. 1994. Manual de métodos de análisis de laboratorio de ecología marina. 13p.

27. HÉQUIETTE, A.; HEMDANE, Y.; ANTHONY, J. 2008. Sediment transport under wave and current combined flows on a tide-dominated shoreface, Northern coast of France. Marine Geology. (Paises Bajos). 249: 226242.

28. INVEMAR. 2003. Informe del estado de los ambientes marinos y costeros de Colombia. serie de publicaciones periódicas; No.8, Santa Marta. (Colombia). 178p.

29. JAIMES, J.; PINZÓN, Y.; TRUJILLO, C. 2004. Explorando alternativas de pesca responsable con la langosta espinosa en Santa Marta. Fundación Sila Kangama, Santa Marta. 80p.

30. LAMAS, N. 2006. Taller color de los sedimentos. Como y porqué. The color of sediments. How and why. Enseñanza de las Ciencias de la Tierra. (España). 14(1):26-28.

31. MARTÍNEZ, J. 1986. Metodologías granulométricas seguimiento de playas. Univ. Politécnica de Las Palmas. Las Palmas. (España). p.1-58.

32. MARTÍNEZ, J.; ADARRAGA, I. 2001. Distribución batimétrica de comunidades macrobentónicas de sus- trato blando en la plataforma continental de Guipúzcoa (golfo de Vizcaya). Bol. Inst. Esp. Oceanogr. (España). 17(1 y 2):33-48.

33. MARTÍNEZ-RAMÍREZ, O.; GRIJALBA-BENDECK, M.; TRUJILLO-ARCILA, C.; ACERO, A. 2009. Primer registro de Liopropoma carmabi (Perciformes, Serranidae) para el Caribe colombiano. Bol. Invest. Mar. Cost. (Colombia). 38(1):205-209.

34. MARTINS, L. 2003. Recent sediments and grain-size analysis. Gravel. (Brasil). 1:90-105.

35. MÉNDEZ, M.; SOLIS-WEISS, V.; CARRANZA-EDWARDS, A. 1986. La importancia de la granulometría en la distribución de organismos bentónicos. Estudio de playas del estado de Veracruz, México. An. Inst. Mar. y Limnol. (México). 13(3):45-56.

36. PÁEZ-OSUNA, F.; FONG-LEE, M.; FERNÁNDEZ-PÉREZ, H. 1984. Comparación de tres técnicas para analizar materia orgánica en sedimentos. An. Inst. Cienc. Mar y Limnol. 11(1):257-264.

37. PARRADO, J.M.; ACHAB, M. 1999. Grain-size trends associated with sediment transport patterns in $\mathrm{Ca}$ diz Bay (southwest Iberian Peninsula). Bol. Inst. Esp. Oceanogr. (España). 15(1-4):269-282.

38. RAMÍREZ-BARÓN, J.; FRANCO-HERRERA, A.; GARCÍA-HOYOS, L.; LÓPEZ, D. 2010. La comunidad fitoplanctónica durante eventos de surgencia y no surgencia, en la zona costera del departamento del Magdalena, Caribe colombiano. Bol. Invest. Mar. Cost. (Colombia). 39(2):233-263.

39. RUÍZ, B.; ILIN, A. 1979. Materia orgánica en los sedimentos de los golfos de Paria y Cariaco, Venezuela. Bol. Inst. Univ. Oriente. (Venezuela). 18(1 y 2):37-52.

40. SALAZAR, J.; ROSAS, J.; RODRÍGUEZ, J. 2003. Condiciones sedimentológicas de la laguna la restinga, isla de Margarita, Venezuela. Interciencia. (Venezuela). 28(1):44-50.

41. SEBENS, K. 1991. Habitat structure and community dynamics in marine benthic systems. En: Bell, S.; Mc Coy, E.; Mushinsky, H.R. Habitat structure: the physical arrangement of objects in space. Chapman and Hall, London. U.K. 438p.

42. WIESER, W. 1969. The effect of grain size on the distribution of small invertebrates inhabiting the beaches 
of puget sond. Limnology and Oceanography. (Estados (Inidos) 4:181-194.

43. VALDÉS, J.; LÓPEZ, L.; LO MÓNACO, S.; ORTLIEB, L. 2000. Condiciones paleoambientales de sedimentación y preservación de materia orgánica en bahía Mejillones del Sur (23 S), Chile. Rev. Biol. Mar. Oceang. (Chile). 35(2):169-180.

44. VEGAS, M. 1971. Introducción a la ecología del bentos marino. Editorial Eva V. Chesneau. Washington, D.C. Estados Unidos. 97p.

45. VISHER, G. 1969. Grain size distribution and depositional processes. J. Sediment. Petrology. (Estados Unidos). 39(3):1074-1106.
46. VITA, R.; MARÍN, J.; MADRID, J.; JIMÉNEZ-BRINQUIS, B.; CÉSAR, A.; MARÍN-GUIRAO, L. 2002. Impacto ambiental de la acuicultura en el bentos marino: experimentos de exclusión-inclusión. Bol. Inst. Esp. Oceanog. (España). 18(1-4):75-86.

47. VON, R. 1976. Contribución al conocimiento del género Voluta LINNÉ, 1758 (Prochobranchia) en la costa del Caribe de Colombia. Mitt. Inst. Colombo-Alemán Invest. Cient. (Colombia) 8: 83-104.

Recibido: Noviembre 30 de 2011

Aceptado: Marzo 15 de 2013 